\title{
Soft masses in SUSY SO(10) GUTs with low intermediate scales
}

\author{
Valentina De Romeri** \\ AHEP Group, Instituto de Física Corpuscular - C.S.I.C./Universitat de València, Edificio de \\ Institutos de Paterna, Apartado 22085, E-46071 València, Spain \\ E-mail: deromeridific.uves
}

\begin{abstract}
The specific shape of the squark, slepton and gaugino mass spectra, if measured with sufficient accuracy, can provide invaluable information not only about the dynamics underpinning their origin at some very high scale such as the unification scale $M_{\mathrm{G}}$, but also about the intermediate scale physics encountered throughout their RGE evolution down to the energy scale accessible for the LHC. In this work, we study general features of the TeV scale soft SUSY breaking parameters stemming from a generic mSugra configuration within certain classes of SUSY SO(10) GUTs with different intermediate symmetries below $M_{\mathrm{G}}$. We show that particular combinations of soft masses show characteristic deviations from the mSugra limit in different models and thus, potentially, allow to distinguish between these, even if the new intermediate scales are outside the energy range probed at accelerators. We also compare our results to those obtained for the three minimal seesaw models with mSugra boundary conditions and discuss the main differences between those and our $S O(10)$ based models.
\end{abstract}

preprint: IFIC/12-11

Proceedings of the Corfu Summer Institute 2011 "School and Workshops on Elementary Particle Physics and Gravity"

September 4-18, 2011

Corfu, Greece

\footnotetext{
* Speaker.

${ }^{\dagger}$ Talk based on [四], done in collaboration with Martin Hirsch and Michal Malinsky.
} 


\section{Introduction}

All proposed supersymmetry (SUSY) breaking schemes have to introduce some high energy scale, where soft terms are generated [乙, [], 团]. SUSY particle masses at the electro-weak (TeV) scale then have to be calculated from the fundamental parameters of the models using renormalization group equations (RGEs). In principle, any superfield beyond the Minimal Supersymmetric extension of the Standard Model (MSSM) - with mass below the SUSY-breaking scale - may be added to the theory. Since the evolution under RGEs is sensitive to the particle content of the theory, any new field will leave its imprint on the soft parameters. Nevertheless, adding new particles beyond the MSSM spectrum can easily spoil the attractive feature that in the MSSM, if SUSY particles have TeV-scale masses, the gauge couplings unify (nearly) perfectly at around $M_{\mathrm{G}} \sim 2 \times 10^{16}$ $\mathrm{GeV}$; thus, the requirement of gauge coupling unification (GCU) imposes a severe constraint on SUSY model building. Here we study soft SUSY-breaking masses within certain classes of SUSY $S O(10)$ theories with different intermediate symmetries below the GUT scale $M_{\mathrm{G}}$. Our main mo-

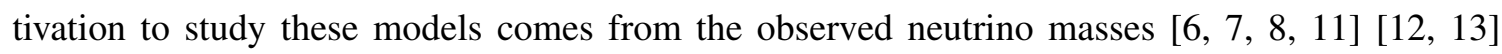

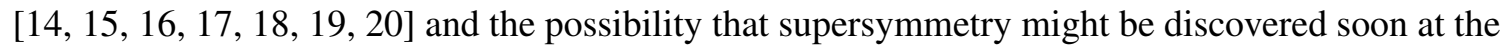
LHC.

Since our main motivation is neutrino masses, we consider models based on $S O(10)$ [23], which automatically contain the necessary ingredients to generate non-zero neutrino masses. All $\mathrm{SO}(10)$ breaking chains [26] of interest to us contain a left-right symmetry (LR) at some stage. In SUSY LR models which use triplets to break $S U(2)_{R}$, [27, [28], 29, [3] ], one can not lower the scale of breaking $\left(v_{R}\right)$ arbitrarily, since one encounters either problems with proton decay or with perturbativity [3]]. However, the situation is different in models with doublets [33]. It is possible to construct models in which the scale of $U(1)_{R} \times U(1)_{B-L}$ breaking, $\mathrm{v}_{\mathrm{BL}}$, can be as low as TeV, or even the full $S U(2)_{R}$ can be brought down to the electro-weak scale [B2], [34].

Within the mSugra framework, one can define certain combinations of soft parameters, which are independent of the high scale input parameters at leading order. We will call such combinations "RGE invariants" [40]. We will construct variants of the models proposed in [33, 34] and will also consider a completely new model, in which $\mathrm{v}_{\mathrm{R}}$ can be brought down to the electro-weak scale with the help of an intermediate Pati-Salam scale [4]]. We will show how the invariants are good indicators to distinguish between different GUT-based SUSY models.

\section{Specific SUSY SO(10) GUT models}

\subsection{General remarks}

In all cases, we demand that the models should be realistic in several basic aspects and potentially interesting for our scope, namely: (i) SUSY SO(10) unification with a sliding intermediate scale by which we mean that the position of a certain intermediate scale can be moved over a large energy range whilst the full compatibility with the electroweak constraints is maintained; (ii) 


\begin{tabular}{|c|c|c|c|}
\hline Field & $\mathrm{m}$ & $3_{c} 2_{L} 2_{R} 1_{B-L}$ & $S O(10)$ \\
\hline$Q$ & 3 & $\left(3,2,1,+\frac{1}{3}\right)$ & 16 \\
$Q^{c}$ & 3 & $\left(\overline{3}, 1,2,-\frac{1}{3}\right)$ & 16 \\
$L$ & 3 & $(1,2,1,-1)$ & 16 \\
$L^{c}$ & 3 & $(1,1,2,+1)$ & 16 \\
$S$ & 3 & $(1,1,1,0)$ & 1 \\
$\delta_{d}, \bar{\delta}_{d}$ & 1 & $\left(3,1,1,-\frac{2}{3}\right),\left(\overline{3}, 1,1,+\frac{2}{3}\right)$ & 10 \\
\hline$\Phi$ & 1 & $(1,2,2,0)$ & 10,120 \\
$\chi, \bar{\chi}$ & 1 & $(1,2,1, \pm 1)$ & $\overline{16}, 16$ \\
$\chi^{c}, \bar{\chi}^{c}$ & 3 & $(1,1,2, \mp 1)$ & $\overline{16}, 16$ \\
\hline
\end{tabular}

Table 1: The relevant part of the field content of Model I with a sliding $S U(2)_{R}$-breaking scale discussed in Sect. 2.2.1.a. In the third column the relevant fields are characterized by their $S U(3)_{c} \times$ $S U(2)_{L} \times S U(2)_{R} \times U(1)_{B-L}$ quantum numbers while their $S O(10)$ origin is specified in the fourth column.

\begin{tabular}{|c|c|c|c|}
\hline Field & $\mathrm{m}$ & $3_{c} 2_{L}{ }_{2} 1_{B-L}$ & $S O(10)$ \\
\hline$Q$ & 3 & $\left(3,2,1,+\frac{1}{3}\right)$ & 16 \\
$Q^{c}$ & 3 & $\left(\overline{3}, 1,2,-\frac{1}{3}\right)$ & 16 \\
$L$ & 3 & $(1,2,1,-1)$ & 16 \\
$L^{c}$ & 3 & $(1,1,2,+1)$ & 16 \\
$S$ & 3 & $(1,1,1,0)$ & 1 \\
$\delta_{u}, \bar{\delta}_{u}$ & 1 & $\left(3,1,1,+\frac{4}{3}\right),\left(\overline{3}, 1,1,-\frac{4}{3}\right)$ & 45 \\
\hline$\Phi$ & 1 & $(1,2,2,0)$ & 10,120 \\
$\chi, \bar{\chi}$ & 1 & $(1,2,1, \pm 1)$ & $\overline{16}, 16$ \\
$\chi^{c}, \bar{\chi}^{c}$ & 2 & $(1,1,2, \mp 1)$ & $\overline{16}, 16$ \\
\hline
\end{tabular}

Table 2: The same as in TABLE I for Model II defined in Sect. 2.2.1.b. The main variation with respect to Model $\mathrm{I}$ is the $B-L$ charge of the vector-like colour triplet pair owing to its different $S O(10)$ origin. In order to maintain the MSSM-like unification pattern, the number of the $S U(2)_{R}$ doublets has been reduced.

Renormalizable $S O(10) \rightarrow$ MSSM gauge symmetry breaking ; (iii) Potentially realistic fermionic spectra ; (iv) MSSM Higgs doublet structure suitable for the implementation of the standard radiative symmetry breaking and also as a means to get unrelated Yukawa couplings for quarks and charged leptons. As to the Requirement 1 above, we shall be namely interested in SUSY SO(10) models with a sliding $S U(2)_{R}$ breaking scale which would be assumed to range from as low as several TeV up to essentially the GUT scale. Such models (e.g. [B4], c.f., Model I and Model II in section 2.2) can be devised either by the introduction of other multiplets, thus leading to RGE invariants which can be strongly model-specific, or by invoking an extra intermidiate scale.

The sensitivity to the intermediate-scales dynamics should be even more pronounced in the latter class of scenarios with more than a single such scale at play. This feature is going to be clearly visible in the specific model of this kind, c.f., Model III in section 2.2 .

However, a strong dependence of the invariants on the sliding scale should not be viewed as a generic feature of the SUSY SO(10) GUTs. Indeed, there are simple scenarios in which the sliding intermediate scale does leave almost no imprints in the soft spectrum. We shall demonstrate this on a specific model with a sliding intermediate $U(1)_{B-L}$ scale of the kind given in [33], c.f., Model IV in the section [2.2.

\subsection{SUSY $S O(10)$ models with a sliding $S U(2)_{R}$ scale}

\subsubsection{Models I and II: single sliding intermediate scale}

First, we shall introduce two variants of the model advocated in [34] which supply the original setting with a few extra ingredients in order to make it potentially realistic, c.f., Sect.

Model I: The field content relevant to the running in this Model is specified in TABLE $\square$. 
The original $S O(10)$ gauge symmetry is broken down to the MSSM in two steps via an intermediate $S U(3)_{c} \times S U(2)_{L} \times S U(2)_{R} \times U(1)_{B-L}$ symmetry stage. The $b_{i}$-coefficients at the $S U(3)_{c} \times S U(2)_{L} \times S U(2)_{R} \times U(1)_{B-L}$ level read $b_{3}=-2, b_{L}=2, b_{R}=4$ and $b_{B-L}^{\mathrm{c}}=13$ where the last number corresponds to the canonically normalized $B-L$ charge, which is obtained from the "physical" one (based on $B_{Q}^{\mathrm{p}}=+\frac{1}{3}$ and $L_{L}^{\mathrm{p}}=+1$ ) by means of the formula $(B-L)^{\mathrm{c}}=\sqrt{\frac{3}{8}}(B-L)^{\mathrm{p}}$. The scale of the $S U(2)_{R} \times U(1)_{B-L}$ breaking is not determined because it drops from the formula for the unification scale (owing namely to the hypercharge-matching condition $\alpha_{Y}^{-1}=\frac{3}{5} \alpha_{R}^{-1}+$ $\left.\frac{2}{5} \alpha_{B-L}^{-1}\right)$ and affects only the value of the GUT-scale gauge coupling $\alpha_{G}$ which, however, is subject of much weaker constraints. More precisely, consider the RGE master equation:

$$
\alpha_{i}^{-1}\left(\mathrm{t}_{\mathrm{a}}\right)=\alpha_{\mathrm{i}}^{-1}\left(\mathrm{t}_{\mathrm{b}}\right)+\frac{\mathrm{b}_{\mathrm{i}}^{\mathrm{a}-\mathrm{b}}}{2 \pi}\left(\mathrm{t}_{\mathrm{b}}-\mathrm{t}_{\mathrm{a}}\right)
$$

where $\mathrm{i}=\mathrm{Y}, \mathrm{R}, \mathrm{B}-\mathrm{L}, 3, t_{X}$ stands for $\ln \left(\mathrm{v}_{\mathrm{X}}\right), b_{i}^{a-b}$ are the b-coefficients in the regime $\left[\mathrm{t}_{\mathrm{a}}, \mathrm{t}_{\mathrm{b}}\right]$ and $\mathrm{t}_{\mathrm{a}}<$ $t_{b}$. The values of the coupling constants at the $m_{Z}$ scale are known [35]. Let us use a matrix notation to specify the gauge couplings $C_{X}$. At the GUT scale for instance, $C_{0}=\operatorname{diag}\left(\alpha_{\mathrm{GUT}}^{-1}, \alpha_{\mathrm{GUT}}^{-1}, \alpha_{\mathrm{GUT}}^{-1}, \alpha_{\mathrm{GUT}}^{-1}\right)$. Define also $\gamma_{R}=\operatorname{diag}\left(\mathrm{b}_{\mathrm{B}-\mathrm{L}}^{\mathrm{R}}, \mathrm{b}_{\mathrm{L}}^{\mathrm{R}}, \mathrm{b}_{3}^{\mathrm{R}}, \mathrm{b}_{\mathrm{R}}^{\mathrm{R}}\right)$ in the first regime $\left[\mathrm{v}_{\mathrm{R}}, \mathrm{v}_{\mathrm{GUT}}\right]$ and $\gamma_{M S S M}=\operatorname{diag}\left(\mathrm{b}_{\mathrm{L}}^{\mathrm{MSSM}}, \mathrm{b}_{3}^{\mathrm{MSSM}}, \mathrm{b}_{\mathrm{Y}}^{\mathrm{MSSM}}\right)$ in the second regime $\left[\mathrm{v}_{\mathrm{SUSY}}, \mathrm{v}_{\mathrm{R}}\right]$. Finally, the $\mathrm{v}_{\mathrm{R}}$ matching condition is contained in

$$
p=\left(\begin{array}{cccc}
1 & 0 & 0 & 0 \\
0 & 1 & 0 & 0 \\
0 & 0 & \sqrt{3 / 5} & \sqrt{2 / 5}
\end{array}\right) .
$$

Then, in the three regimes $\left[\mathrm{m}_{\mathrm{Z}}, \mathrm{v}_{\mathrm{SUSY}}\right],\left[\mathrm{v}_{\mathrm{SUSY}}, \mathrm{v}_{\mathrm{R}}\right],\left[\mathrm{v}_{\mathrm{R}}, \mathrm{v}_{\mathrm{GUT}}\right]$, the one-loop gauge coupling running is described by:

$$
\begin{aligned}
C_{R} & =C_{0}+\gamma_{R}\left(\mathrm{t}_{\mathrm{GUT}}-\mathrm{t}_{\mathrm{R}}\right) /(2 \pi) ; \\
C_{M S S M} & =p \cdot C_{R} \cdot p^{T}+\gamma_{M S S M}\left(\mathrm{t}_{\mathrm{R}}-\mathrm{t}_{\mathrm{SUSY}}\right) /(2 \pi) ; \\
\alpha_{i}\left(\mathrm{t}_{\mathrm{SUSY}}\right) & =\alpha_{i}\left(\mathrm{~m}_{\mathrm{Z}}\right)-\mathrm{b}_{\mathrm{i}}^{\mathrm{SM}}\left(\mathrm{t}_{\mathrm{SUSY}}-\mathrm{m}_{\mathrm{Z}}\right) /(2 \pi) .
\end{aligned}
$$

Equate $\alpha_{2}^{-1}\left(t_{G U T}\right)=\alpha_{R}^{-1}\left(t_{G U T}\right)$ expressed in terms of the known values at the $\mathrm{Z}$ scale to get the GUT scale:

$$
\begin{gathered}
t_{G U T}=1 /\left(3 b_{R}^{R}-5 b_{L}^{R}+2 b_{B-L}^{R}\right)\left((10 \pi)\left(\alpha_{B-L}\left(m_{Z}\right)-\alpha_{L}\left(m_{Z}\right)\right]\right) \\
+t_{\mathrm{R}}\left(2 b_{\mathrm{B}-\mathrm{L}}^{\mathrm{R}}-5 \mathrm{~b}_{\mathrm{L}}^{\mathrm{R}}+3 \mathrm{~b}_{\mathrm{R}}^{\mathrm{R}}+5 \mathrm{~b}_{\mathrm{L}}^{\mathrm{MSSM}}-5 \mathrm{~b}_{\mathrm{B}-\mathrm{L}}^{\mathrm{MSSM}}\right) \\
+5 \mathrm{t}_{\mathrm{SUSY}}\left(\mathrm{b}_{\mathrm{L}}^{\mathrm{SM}}-\mathrm{b}_{\mathrm{Y}}^{\mathrm{SM}}-\mathrm{b}_{\mathrm{L}}^{\mathrm{MSSM}}+\mathrm{b}_{\mathrm{Y}}^{\mathrm{MSSM}}\right) \\
\left.+5 \mathrm{t}_{\mathrm{Z}}\left(\mathrm{b}_{\mathrm{Y}}^{\mathrm{SM}}-\mathrm{b}_{\mathrm{L}}^{\mathrm{SM}}\right)\right) .
\end{gathered}
$$

It can be easily checked that the coefficient in front of the $t_{R}$ is zero. This, makes the gauge coupling unification in Model I qualitatively similar to the MSSM case, see FIG. W, also in the non-exact unification.

In what follows, we shall simply parametrize our ignorance of the shape of the GUT spectrum by considering unification regions from where the $S U(3)_{C} \times S U(2)_{L} \times S U(2)_{R} \times U(1)_{B-L}$ gauge couplings can emanate rather than unique unification points, c.f., FIG. 团 and discussion in Sect. $4 . \mathbb{D}$. 

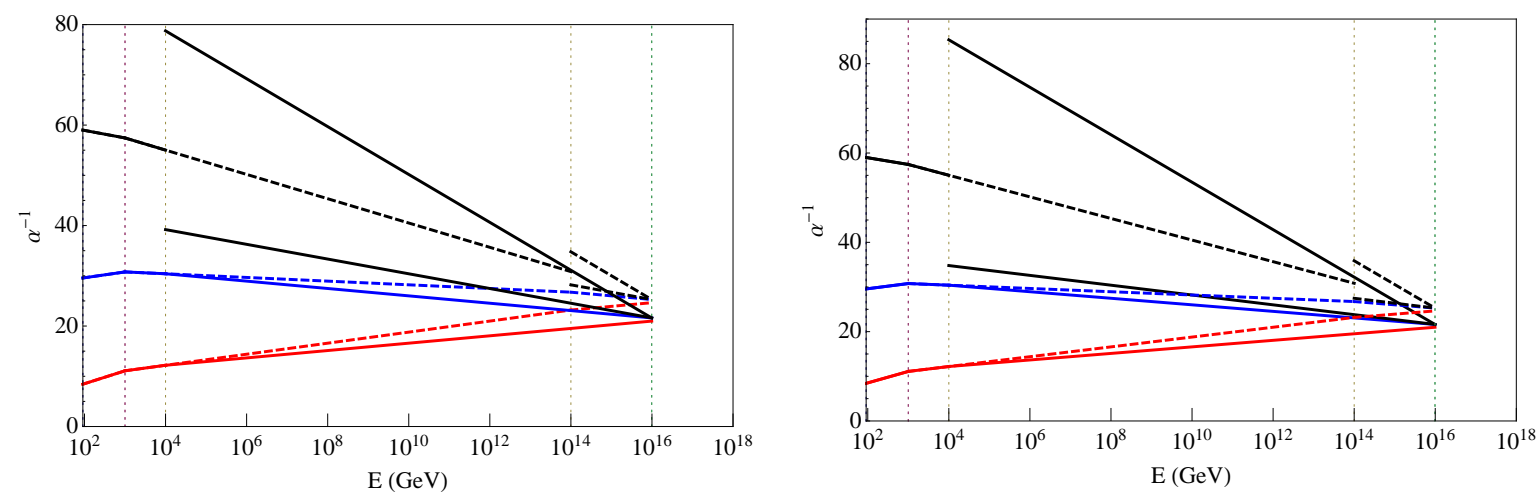

Figure 1: Gauge coupling unification in Model I (left) and in Model 2 (right) in two limits corresponding to different positions of the sliding $S U(2)_{R} \times U(1)_{B-L}$ breaking scale $\mathrm{v}_{\mathrm{R}}$. In solid lines, we depict the RGE behaviour of the gauge couplings for $\mathrm{v}_{\mathrm{R}}$ in the vicinity of the electroweak scale $\mathrm{v}_{\mathrm{R}} \sim 10^{4} \mathrm{GeV}$ while the dashed lines correspond to $\mathrm{v}_{\mathrm{R}} \sim 10^{14} \mathrm{GeV}$. The position of the intersection region shifts slightly up with rising $\mathrm{v}_{\mathrm{R}}$ but the corresponding scale remains intact.

Model II: The relevant $b_{i}$-coefficients at the $S U(3)_{c} \times S U(2)_{L} \times S U(2)_{R} \times U(1)_{B-L}$ level read $b_{3}=-2, b_{L}=2, b_{R}=3$ and $b_{B-L}^{\text {can }}=29 / 2$. Indeed, these numbers differ from Model I only in the $S U(2)_{R} \times U(1)_{B-L}$ sector and the variations in the relevant $b$-coefficients obey $\Delta b_{R}+\frac{2}{3} \Delta b_{B-L}=0$ so the $b$-coefficient associated to the "effective" MSSM hypercharge is the same as in Model I. Nevertheless, as we shall see in Sect. 4.], even such a slight change in the gauge-coupling behaviour at the $S U(3)_{c} \times S U(2)_{L} \times S U(2)_{R} \times U(1)_{B-L}$ level is enough to generate a significant difference between the Model-I and Model-II soft invariants, especially if the $S U(3)_{c} \times S U(2)_{L} \times S U(2)_{R} \times$ $U(1)_{B-L}$ running is long. However, if the $S U(2)_{R} \times U(1)_{B-L}$ gauge symmetry happens to be broken close to the GUT scale, the two models will be indistinguishable from the soft-sector point of view.

\subsubsection{Model III: sliding $S U(2)_{R}$ and Pati-Salam scales}

The third model of our interest belongs to the second category of the simple classification given in Sect. [.]. In particular, the sliding nature of the $S U(2)_{R} \times U(1)_{B-L}$ scale is achieved via an interplay with another intermediate scale, namely, the Pati-Salam $S U(4)_{C} \times S U(2)_{L} \times S U(2)_{R}$. Thus, the initial $S O(10)$ gauge symmetry is broken down to the MSSM in three steps. The field content relevant to the two intermediate-symmetry stages is given in TABLE 1$]$.

At the Pati-Salam stage, the $b_{i}$-coefficients read $b_{4}=3, b_{L}=6, b_{R}=14$ while at the $S U(3)_{c} \times$ $S U(2)_{L} \times S U(2)_{R} \times U(1)_{B-L}$ level they are $b_{3}=-2, b_{L}=3, b_{R}=11$ and $b_{B-L}^{\text {can }}=10$.

In this model, both the position of the GUT scale as well as the value of $\alpha_{G}$ depend on both intermediate scales. However, unlike in Models I and II, here the gauge unification can always be made exact, c.f., FIG. \, even at the one-loop level, and, thus, there is no extra theoretical uncertainty other than the error in the electroweak-scale $\alpha_{s}$ to be taken into account.

\subsection{SUSY $S O(10)$ models with a sliding $U(1)_{R}$ scale}

The full $S U(2)_{R}$ is not the minimal option to realize a gauge symmetry acting in the RH sector 


\begin{tabular}{|c|c|c|c|c|}
\hline Field & $\mathrm{m}$ & $3_{c} 2_{L} 2_{R} 1_{B-L}$ & Pati-Salam & $S O(10)$ \\
\hline$Q$ & 3 & $\left(3,2,1,+\frac{1}{3}\right)$ & $(4,2,1)$ & 16 \\
$Q^{c}$ & 3 & $\left(\overline{3}, 1,2,-\frac{1}{3}\right)$ & $(\overline{4}, 1,2)$ & 16 \\
$L$ & 3 & $(1,2,1,-1)$ & $(4,2,1)$ & 16 \\
$L^{c}$ & 3 & $(1,1,2,+1)$ & $(\overline{4}, 1,2)$ & 16 \\
$\Sigma^{c}$ & 3 & $(1,1,3,0)$ & $(1,1,3)$ & 45 \\
$\delta_{d}, \bar{\delta}_{d}$ & 1 & $\left(3,1,1, \mp \frac{2}{3}\right)$ & $(6,1,1)$ & 10 \\
\hline$\Phi$ & 2 & $(1,2,2,0)$ & $(1,2,2)$ & 10 \\
$\Omega$ & 1 & $(1,1,3,0)$ & $(1,1,3)$ & 45 \\
$\chi, \bar{\chi}$ & 1 & $(1,2,1, \pm 1)$ & $(\overline{4}, 2,1),(4,2,1)$ & $\overline{16}, 16$ \\
$\chi^{c}, \bar{\chi}^{c}$ & 1 & $(1,1,2, \mp 1)$ & $(4,1,2),(\overline{4}, 1,2)$ & $\overline{16}, 16$ \\
$\Psi$ & 1 & absent & $(15,1,1)$ & 45 \\
\hline
\end{tabular}

\begin{tabular}{|c|c|c|c|c|}
\hline Field & $\mathrm{m}$ & $3_{c} 2_{L} 1_{R} 1_{B-L}$ & $3_{c} 2_{L} 2_{R} 1_{B-L}$ & $S O(10)$ \\
\hline$Q$ & 3 & $\left(3,2,0,+\frac{1}{3}\right)$ & $\left(3,2,1,+\frac{1}{3}\right)$ & 16 \\
$Q^{c}$ & 3 & $\left(\overline{3}, 1, \pm \frac{1}{2},-\frac{1}{3}\right)$ & $\left(\overline{3}, 1,2,-\frac{1}{3}\right)$ & 16 \\
$L$ & 3 & $(1,2,0,-1)$ & $(1,2,1,-1)$ & 16 \\
$L^{c}$ & 3 & $\left(1,1, \pm \frac{1}{2},+1\right)$ & $(1,1,2,+1)$ & 16 \\
$S$ & 3 & $(1,1,0,0)$ & $(1,1,1,0)$ & 1 \\
\hline$\Phi$ & 2 & $\left(1,2, \pm \frac{1}{2}, 0\right)$ & $(1,2,2,0)$ & 10 \\
$\Omega$ & 1 & absent & $(1,1,3,0)$ & 45 \\
$\chi, \bar{\chi}$ & 1 & absent & $(1,2,1, \pm 1)$ & $\overline{16}, 16$ \\
$\chi^{c}, \bar{\chi}^{c}$ & 1 & $\left(1,1, \pm \frac{1}{2}, \mp 1\right)$ & $(1,1,2, \mp 1)$ & $\overline{16}, 16$ \\
\hline
\end{tabular}

Table 3: The effective field contents of Model III in the two intermediate symmetry stages.

Table 4: The effective field contents of Model IV relevant to the two intermediate symmetry stages.
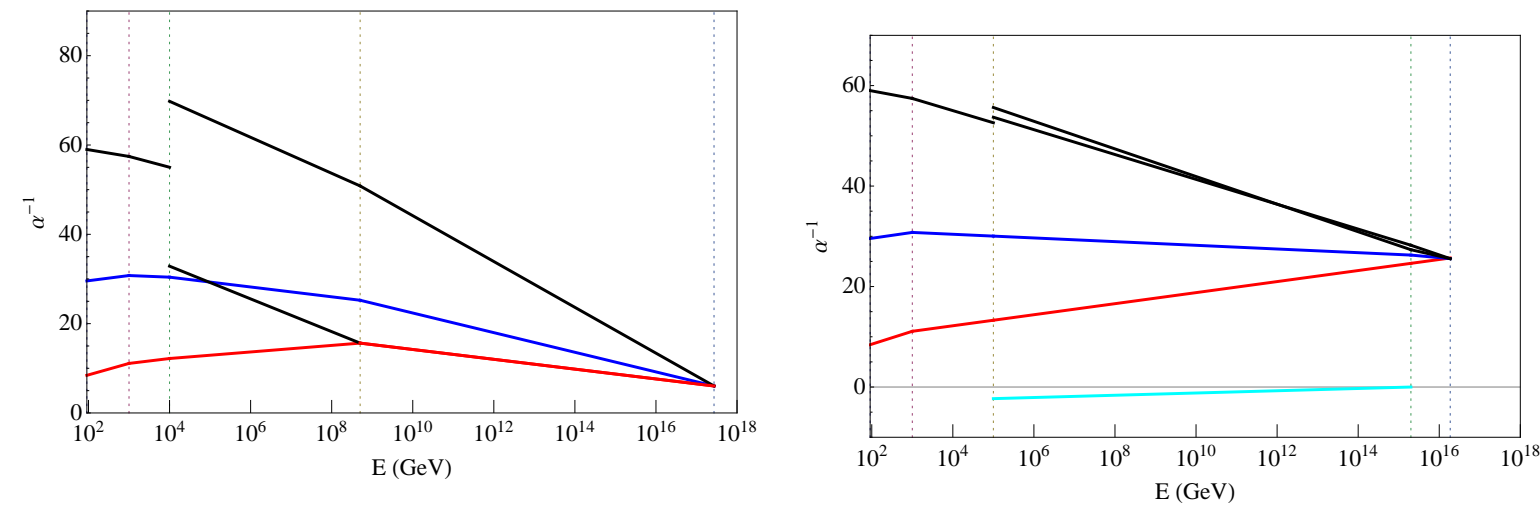

Figure 2: Running in the Model III variant of the low-LR scale SUSY SO(10). The value of $\mathrm{v}_{\mathrm{PS}}$ is correlated to the position of the L-R breaking scale $v_{R}$ which can again slide from as low as few $\mathrm{TeV}$ up to roughly $10^{14} \mathrm{GeV}$, c.f., FIG. 8 .

Figure 3: Running in Model IV with a low $B-L$ scale. Note the effects of the U(1) mixing in the running \& matching; the lowest curve corresponds to the off-diagonals of the $\left(G G^{T} / 4 \pi\right)^{-1}$ matrix.

of the matter spectrum in a way compatible with the MSSM quantum numbers: the hypercharge sum-rule $Y=T_{R}^{3}+(B-L) / 2$ trivially holds even if one sticks to the $U(1)_{R}$ subgroup.

The simplicity of the minimally fine-tuned $U(1)_{R} \times U(1)_{B-L} \rightarrow U(1)_{Y}$ scenarios automatically implies the scale of this spontaneous symmetry breakdown is a sliding one. Thus, the specific position of the $U(1)_{R} \times U(1)_{B-L} \rightarrow U(1)_{Y}$ breaking scale is, in this case, irrelevant for the oneloop gauge running.

This, however, is not the case for the leading-log soft RGE invariants of our interest. In particular, unlike $B_{Y}$, both the $U(1)_{R} \times U(1)_{B-L}$ gauge bosons enter the renormalized propagators of squarks and sleptons and one can expect a residual dependence of the invariants on the $U(1)_{R} \times$ $U(1)_{B-L}$-breaking scale. Nevertheless, as we shall demonstrate in a particular realization of this 
simple scheme, such effects should be much milder than those in the scenarios with the full gauged $S U(2)_{R}$ symmetry.

\subsubsection{Model IV: $U(1)_{R} \times U(1)_{B-L} \rightarrow U(1)_{Y}$ breaking}

Here we consider a variant of the basic SUSY SO(10) model advocated in [33] in which an extended intermediate $U(1)_{R} \times U(1)_{B-L}$ stage follows a short $S U(2)_{R} \times U(1)_{B-L}$ phase. The field content relevant to the $\mathrm{RG}$ running in the first two parts of the symmetry-breaking chain is given in TABLE $⿴$.

The relevant $b_{i}$-coefficients at the $S U(3)_{c} \times S U(2)_{L} \times S U(2)_{R} \times U(1)_{B-L}$ level read $b_{3}=-3$, $b_{L}=2, b_{R}=5$ and $b_{B-L}^{\text {can }}=15 / 2$. In the $S U(3)_{c} \times S U(2)_{L} \times U(1)_{R} \times U(1)_{B-L}$ stage, however, the effects of the $U(1)$ mixing must be taken into account and, thus, the $b$-coefficients in the $U(1)_{R} \times$ $U(1)_{B-L}$ sector constitute a matrix of anomalous dimensions $\gamma$. One has $b_{3}=-3, b_{L}=1$ and

$$
\gamma^{\text {phys }}=\left(\begin{array}{cc}
15 / 2 & -1 \\
-1 & 18
\end{array}\right)
$$

which should be brought into the canonical basis by means of a normalization matrix $N=\operatorname{diag}(1, \sqrt{3 / 8})$, $\gamma^{\text {can }}=N \gamma^{\text {phys }} N$. The details of the one-loop RGE evolution of gauge couplings and soft masses in theories with more than a single abelian gauge factor are summarized in Appendix A of [W]. The qualitative features of the gauge-coupling running in this setting can be seen in FIG. B.

\section{Leading-log RGE invariants}

In this section we focus on the calculation of the invariants using mSugra boundary conditions.

Barring for the moment the effects of $U(1)$ mixing in the renormalization group equations present in Model IV, at the 1-loop level, one can devise a simple set of analytic equations for the soft terms. Gaugino masses scale as gauge couplings do and so the requirement of GCU fixes the gaugino masses at the low scale

$$
M_{i}\left(m_{S U S Y}\right)=\frac{\alpha_{i}\left(m_{S U S Y}\right)}{\alpha\left(M_{G}\right)} M_{1 / 2}
$$

Eq. (13) implies that the relationship of the $M_{i}$ to $M_{1 / 2}$ is changed in Models I to III, since $\alpha\left(M_{G}\right)$ is shifted. Neglecting the Yukawa couplings for the soft mass parameters of the first two generations of sfermions one can write

$$
m_{\tilde{f}}^{2}=m_{0}^{2}+\frac{M_{1 / 2}}{\alpha\left(M_{\mathrm{G}}\right)^{2}} \sum_{R_{j}} \sum_{i=1}^{N} \tilde{f}_{i}^{R} \alpha_{i}\left(v_{R_{j}}\right)^{2} .
$$

Here, the sum over " $R_{j}$ " runs over the different regimes in the models under consideration, while the sum over $i$ runs over all gauge groups in a given regime. $\alpha_{i}\left(v_{R_{j}}\right)$ is to be understood as the gauge coupling of group $i$ evaluated at the upper end of regime $R_{j}$. 
The different $\tilde{f}_{i}^{R}$ can be written in a compact form as:

$$
\tilde{f}_{i}^{R}=\frac{c_{i}^{f, R}}{b_{i}}\left[1-\left(\frac{\alpha_{i}\left(v_{x}\right)}{\alpha_{i}\left(v_{y}\right)}\right)^{2}\right],
$$

where $v_{x}$ and $v_{y}$, respectively, indicate the value of the relevant $\alpha$ at the lower and higher boundaries of the regime under consideration. The $c_{i}^{f, R}$ coefficients given in TABLE V of [四] are proportional to the values of the quadratic Casimir of representation $R_{f}$ hosting the matter field $f$ with respect to the group $G$ in the regime $R: c_{i}^{f, R}=2 C_{G}\left(R_{f}\right)$. Note that the coefficients $c_{i}^{f, R}$ are different for the different fermions, which leads to a different coefficient in front of $M_{1 / 2}$ in eq. (B.2). The $b_{i}$ in eq. (B.3) are the one-loop $b$-coefficients for the different models defined in the previous section. For completeness, the well-known one-loop beta-coefficients for the MSSM are (in the traditional $S U(5)$ normalization): $b=\left(b_{1}, b_{2}, b_{3}\right)^{M S S M}=\left(\frac{33}{5}, 1,-3\right)$.

Individual SUSY masses depend strongly on the initial values for $m_{0}$ and $M_{1 / 2}$. However, one can form four different combinations, which we choose to be

$$
L E \equiv\left(m_{\tilde{L}}^{2}-m_{\tilde{E}}^{2}\right) / M_{1}^{2}, Q E \equiv\left(m_{\tilde{Q}}^{2}-m_{\tilde{E}}^{2}\right) / M_{1}^{2}, D L \equiv\left(m_{\tilde{D}}^{2}-m_{\tilde{L}}^{2}\right) / M_{1}^{2}, Q U \equiv\left(m_{\tilde{Q}}^{2}-m_{\tilde{U}}^{2}\right) / M_{1}^{2} .
$$

It is easy to see that, at the leading-log level, $m_{0}$ and $M_{1 / 2}$ drop out of the equations for the invariants. Note, that one could have equally well normalized to any of the other two gaugino masses. The choice of $M_{1}$ is only motivated by the expectation that it will be the gaugino parameter measured with the smallest error.

\section{Sliding scale imprints in the leading-log RGE Invariants}

\subsection{Models I and II with a sliding $S U(2)_{R}$ scale}

The method: As we have already mentioned in Sect. \, in Models I and II the sliding nature of the $S U(2)_{R}$ scale makes it impossible to get an exact unification, in full analogy with the MSSM. Since, however, this is just about a $2 \%$ effect, we shall not attempt to improve on this by either looking for a suitable set of threshold corrections or by going beyond the one-loop approximation ${ }^{1}$. Rather than that, we shall just parametrize our ignorance of the "true values" of the unification scale position and the unified gauge coupling in terms of a pair of small "offset" parameters scanning over the area of the relevant "non-unification triangle" shown in FIG.(田). In what follows, we shall to use the error on $\alpha_{S}\left(M_{Z}\right)$ given in [48], $\Delta\left(\alpha_{S}\left(M_{Z}\right)\right)=0.002$, which does not take into account the latest QCD lattice calculations results.

The results: In FIGs. $\square$ and 1 we display the $v_{R}$-dependence of the RGE invariants in Models I and II due to the running effects subsumed by Eq. (B.2). The bands correspond to the error in the gauge-coupling unification inherent to these settings which, at the leading-log level, can be taken into account by scanning over the area of the relevant non-unification triangle, c.f., FIG. $\mathbb{\text { t. }}$

\footnotetext{
${ }^{1}$ Indeed, this would be inconsistent as we are concerned only with the leading-log approximation for the softs.
} 


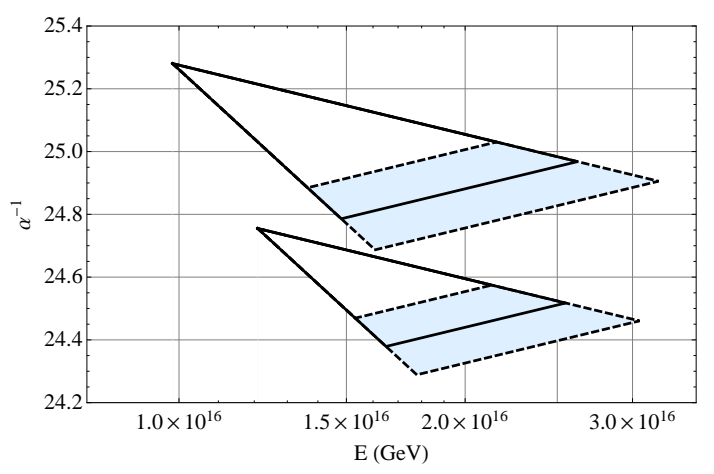

Figure 4: The MSSM-like non-unification triangle in Models I and III with $v_{R}=10^{14} \mathrm{GeV}$ for two different values of the unknown soft-SUSY breaking scale ( $m_{\mathrm{SUSY}}=1 \mathrm{TeV}$ for the upper one and $m_{\mathrm{SUSY}}=500 \mathrm{GeV}$ for the lower). The upper sides of the triangles corresponds to $\alpha_{L}^{-1}$ while the lower-left sides depict the "effective" $\alpha_{Y}^{-1}$ defined as $\frac{3}{5} \alpha_{R}^{-1}+\frac{2}{5} \alpha_{B-L}^{-1}$. The light blue area surrounding the $\alpha_{S}^{-1}$ line represents the $1 \sigma$ uncertainty in $\alpha_{s}\left(M_{Z}\right)$ as given in [4]]. Both triangles move down for lower values of $v_{R}$, see FIGs. 1 and 2.

The upper (yellow) band refers to the combination $\mathrm{QE}$, the (blue) band which at low $\mathrm{v}_{\mathrm{R}}$ partially overlaps with QE represents DL, whereas the third (brown) band is QU and, finally, the lowest (green) band refers to the LE combination. Note that, for practical reasons, the invariants QE and DL have been scaled down by a factor of ten. The same colour-code is adopted in the other figures in this section.

In general, the invariants exhibit a logarithmic dependence on $\mathrm{v}_{\mathrm{R}}$. For $v_{R}$ close to the MSSM scale (on the left), the QU and LE invariants overlap. This is attributed to the enhanced gauge symmetry throughout the whole $m_{\mathrm{SUSY}}-M_{\mathrm{G}}$ range which makes $m_{\tilde{Q}}^{2}$ and $m_{\tilde{U}}^{2}$ as well as $m_{\tilde{L}}^{2}$ and $m_{\tilde{E}}^{2}$ behave the same, see the LR-stage $c_{i}^{\tilde{f}}$-coefficients. In the $\mathrm{v}_{\mathrm{R}} \rightarrow \mathrm{M}_{\mathrm{G}}$ limit, the mSugra values of the invariants (modulo the MSSM non-unification) are reproduced. Concerning QE and DL, the first thing to notice is that these invariants tend to increase with $v_{R}$ departing from $M_{\mathrm{G}}$, thus leading to a pattern characteristic to this class of models. Moreover, they are more sensitive to the initial condition because the colour-effects in their evolution do not cancel, thus leading to larger bands.

Naturally, the main difference between FIG. $\mathbf{G}$ and FIG. $\square$ is expected in the low- $\mathrm{v}_{\mathrm{R}}$ regime where the effects due to the slight difference in the Model-I and Model-II spectra are most pronounced and the QU and LE invariants run faster due to a larger ratio of the coupling constants in the relevant Eq. (B.3)).

\subsection{Model III with sliding $S U(2)_{R}$ and PS scales}

The method: In Model III, the LR and PS intermediate scales can be always adjusted so that one gets an exact one-loop unification for $v_{R}$ stretching up to about $10^{14} \mathrm{GeV}$, c.f., FIG. $\square$. This is technically achieved by relating the value of the PS scale to the value of the LR scale as

$$
t_{P S}=\frac{1}{2} t_{L R}-\frac{1}{12}\left(14 t_{S U S Y}+20 t_{Z}+\pi\left(18 \alpha_{S}\left(t_{Z}\right)^{-1}-33 \alpha_{L}\left(t_{Z}\right)^{-1}+15 \alpha_{Y}\left(t_{Z}\right)^{-1}\right)\right)
$$




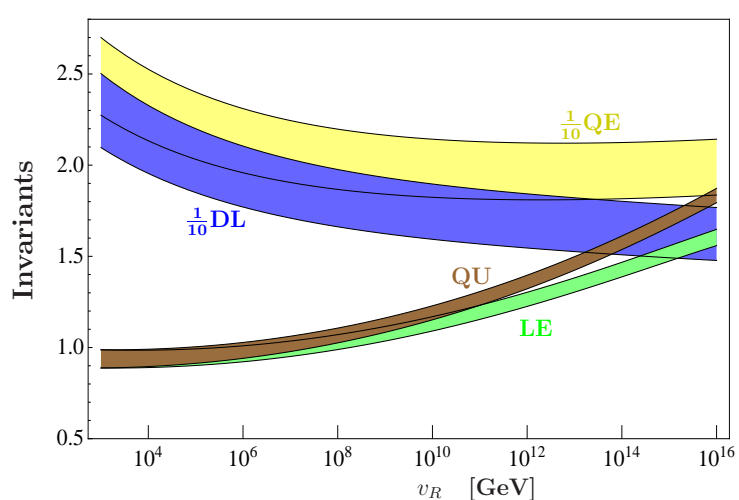

Figure 5: The $v_{R}$-dependence of the leading-log invariants in Model I, c.f., Sect. 2.2.1.a. The bands represent the error due to the non-exact gauge-coupling unification depicted in FIG.5.

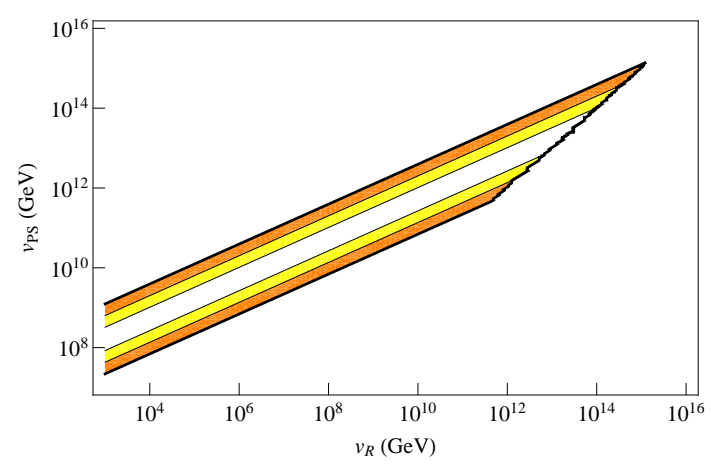

Figure 7: The correlation of the intermediate symmetry-breaking scales in Model III (allowed region coloured). The contours correspond to the quality of the fit of $\alpha_{S}\left(M_{Z}\right)$ for each choice of the Pati-Salam breaking scale $v_{P S}$ an the LR breaking scale $v_{R}$.

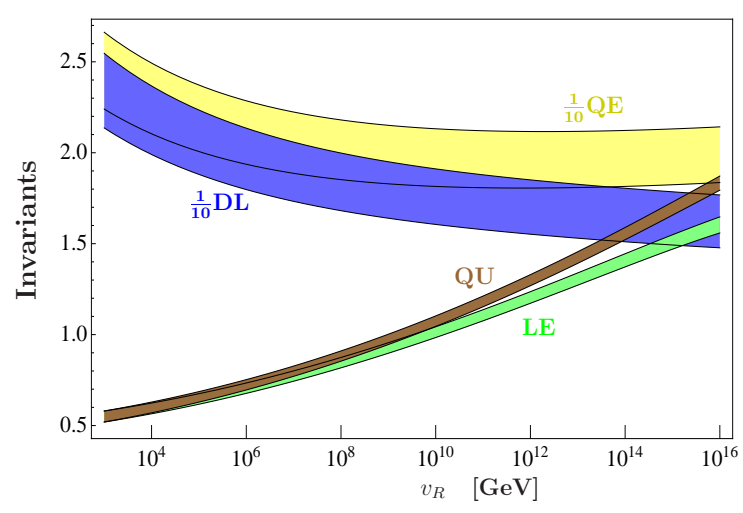

Figure 6: The same as in FIG. 6 but for Model II of Sect. 2.2.1.b. The QU and LE behaviour differs from that in FIG. 6 mainly in the low- $v_{R}$ regime.

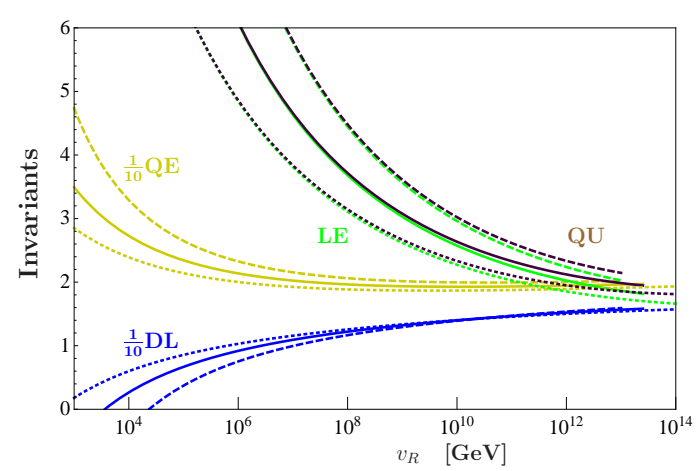

Figure 8: Intermediate-scale dependence of the RGE invariants Model III, see Sect. 2.2.2. For each of the four invariants, the solid curve corresponds to $\alpha_{S}\left(M_{Z}\right)$ fixed at its central value and the dashed and dotted lines refer to the $-1 \sigma$ and $+1 \sigma$ trajectories, respectively; c.f. FIG 7.

Here, the $t_{x}$ stand for $\ln \left(m_{X}\right)$ as usual. Thus, the main uncertainty at this level comes from the experimental error in $\alpha_{S}\left(M_{Z}\right)$. In what follows, we shall vary $v_{R}$ and $v_{P S}$ along the constant $\alpha_{S}\left(M_{Z}\right)$ error trajectories, namely, within $\pm 1 \sigma$, corresponding to the boundaries between the yellow and white areas within the parameter area depicted in FIG. $\square$.

The results: In this case, the intermediate-scale dependence of the leading-log RGE invariants is yet more pronounced than in Models I and II, c.f., FIG 8 . For each of the four invariants, the solid

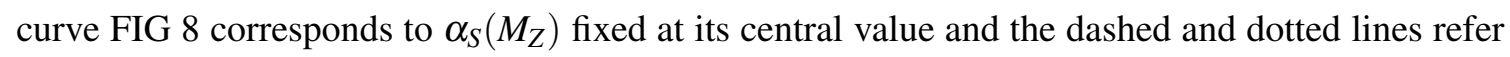
to the $-1 \sigma$ and $+1 \sigma$ trajectories, respectively.

For all four invariants under consideration, we observe a stronger $v_{R}$-dependence than in Mod- 


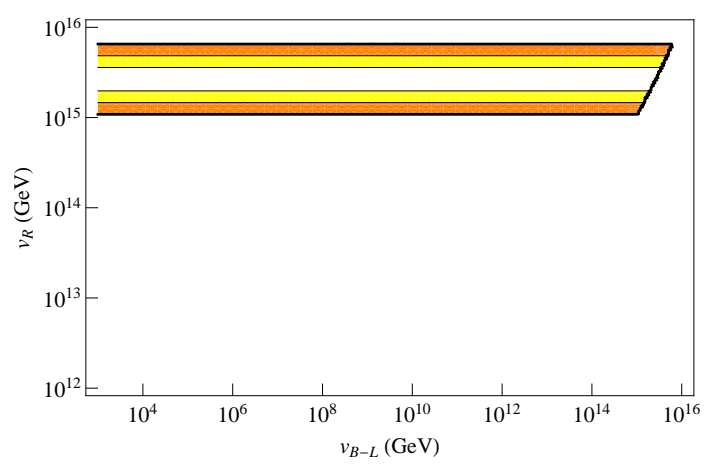

Figure 9: The parameter space of Model IV of Sect. II C. Same legend as in FIG. 7.

els I and II. This is namely due to the extended Pati-Salam running which contributes with larger $c_{i}^{\tilde{f}}$-coefficients than the LR stage. Moreover, unlike in FIGs. $\$$ and $\mathbf{6}$, three out of four invariants grow with lowering $v_{R}$ while the fourth one even becomes negative for $v_{R}$ close to the MSSM scale, thus, again, leading to a very characteristic pattern.

\subsection{Model IV with a sliding $U(1)_{R} \times U(1)_{B-L}$ scale}

The method: Finally, in Model IV, c.f. Sect. [2.3, the unification is exact for any value of the sliding scale $v_{B L}$ below a (constant) $v_{R}$ c.f., FIG. $Q$. Thus, as before, the main uncertainty at this level comes from the experimental error in $\alpha_{S}\left(M_{Z}\right)$ which translates into small shifts in $v_{R}$.

The results: In the two panels of FIG. ㅁ, the four invariants of our interest are depicted as functions of $v_{B L}$. The line legend is the same as in the case of Model III.

Due to the very special nature of the sliding scale in this setting, all four invariants exhibit only a very mild $v_{B L}$ dependence, with the strongest effect of the order of few per cent observed in the LE case. This is because the $v_{B L}$ scale enters into the soft masses only through the slight changes in the abelian gauge couplings, which, however, are overwhelmed by the colour effects in all the other invariants.

\subsection{Squark and slepton spectra.}

In FIG. $\square$ we plot the shapes of the MSSM squark and slepton spectra obtained in mSugra and in Models I, II and III calculated for the SPS3 benchmark point, i.e. for $m_{0}=90 \mathrm{GeV}$ and $M_{1 / 2}=400 \mathrm{GeV}$. For each of the cases, the horizontal lines (bottom to up) correspond to $m_{\tilde{e}^{c}}$ (light blue), $m_{\tilde{l}}$ (blue), $m_{\tilde{u}^{c}}$ (orange), $m_{\tilde{d}^{c}}$ (light orange) and $m_{\tilde{q}}$ (purple). In order to pronounce the differences, the $\mathrm{v}_{\mathrm{R}}$ scale has been in all cases chosen very low, namely, $v_{R} \sim 10^{3} \mathrm{GeV}$, and consequently $v_{P S}$ in Model III is fixed to $v_{P S} \sim 10^{7} \mathrm{GeV}$ by gauge unification. The masses of the $\tilde{d}^{c}$ and of the $\tilde{u}^{c}$ almost coincide in all the Models. Models I and II differ from the mSugra case namely by the smaller splittings observable in the squark as well as in the slepton masses, which is 

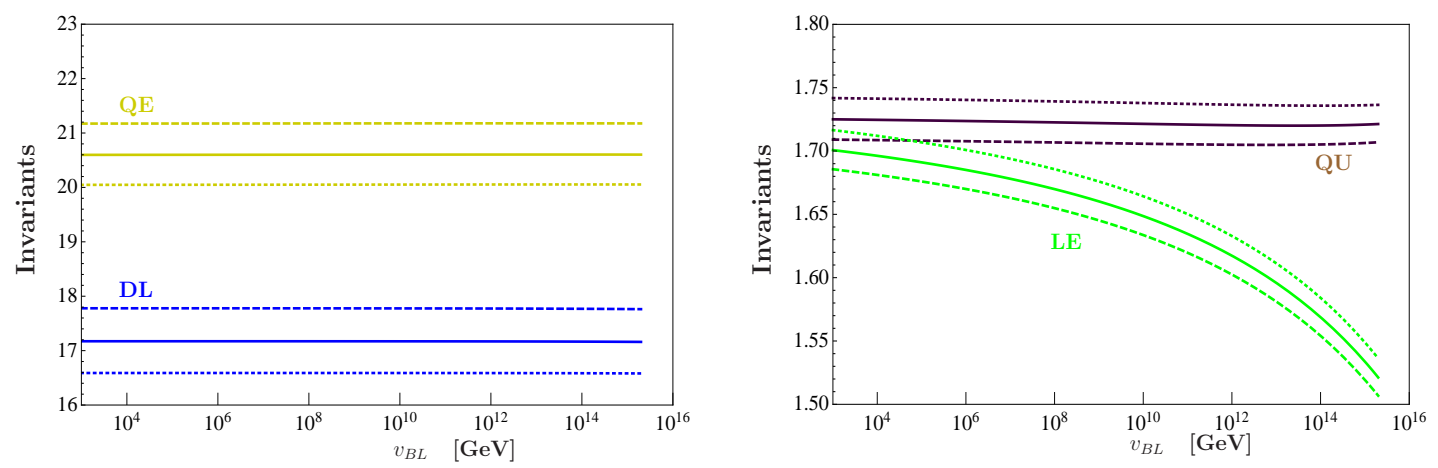

Figure 10: The $v_{B L}$-scale dependence of the RGE invariants in Model IV. For each of the four invariants, the solid curves correspond to $\alpha_{S}\left(M_{Z}\right)$ fixed at its central value while the dashed and dotted lines refer to the $-1 \sigma$ and $+1 \sigma$ trajectories corresponding to roughly $v_{R} \sim 2 \times 10^{15} \mathrm{GeV}$ and $v_{R} \sim 4 \times 10^{15} \mathrm{GeV}$, respectively; c.f. FIG 9.

more pronounced for the latter model. However, the spectrum of Model III is strongly compressed due to an extended Pati-Salam stage which makes it rather outstanding.

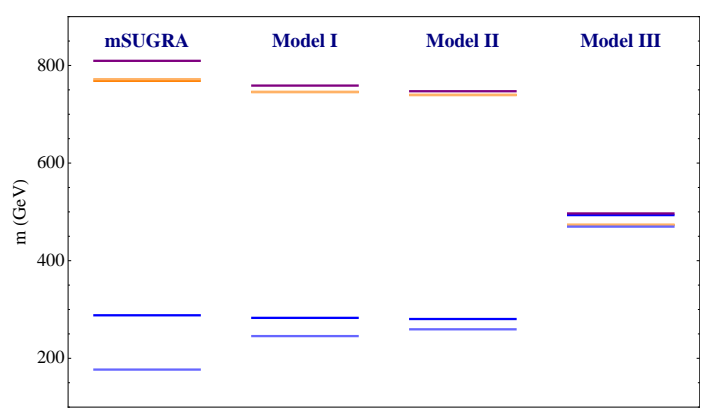

Figure 11: The MSSM squark and slepton spectra mSugra and Models I, II and III calculated for the SPS3 benchmark point We do not show the results for model IV in this figure, since they are very similar to the mSugra case.

\section{Discussion and outlook}

We have studied the leading-log RGE evolution of the MSSM soft SUSY breaking parameters for four different GUT models with mSugra boundary conditions. Although all the settings are based on the unified $S O(10)$ gauge group, they differ at the level of intermediate scale symmetry groups and/or particle content below the GUT scale. All models we consider are able to accommodate the neutrino data by either inverse or linear seesaw.

The extra gauge groups and/or beyond MSSM fields change the evolution of the soft parameters with respect to the basic mSugra expectation. The invariant mass combinations we considered are especially suited to uncover the effects of beyond-mSugra physics on the SUSY spectra. Remarkably, while invariants contain only a logarithmic dependence on the new physics scales, their 
behavior is qualitatively different in different models. In our Models I and II, the invariants LE and QU (c.f., Sect. [1) are always lower than the mSugra limit, while DL and QE are always larger.

In contrast to that, in the Pati-Salam based Model III, LE and QU are always larger than in mSugra, with a rather strong dependence on the $\mathrm{v}_{\mathrm{R}}$ scale, namely due to the higher dimensionality of the relevant multiplets at the Pati-Salam stage. At the same time, in Model III, DL is always below the mSugra limit, while QE hardly varies at all as a function of $v_{R}$. Finally, Model IV is an example of how a new scale can be effectively "hidden" from the RGE invariants in special constructions.

The RGE invariants are, therefore, good model discriminators, at least in principle. Different types of errors need to be considered here. First, there are the errors from uncertainties in the values of the input parameters. The largest error currently stems from the completely unknown $m_{S U S Y}$, see

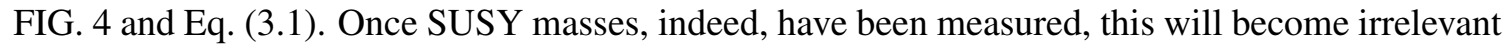
and the largest error will, most likely, be $\Delta\left(\alpha_{S}\right)$.

Next, the RGE invariants considered here are calculated to the leading-log precision only. However, in some cases, important higher order effects such as genuine 2-loop corrections and 1-loop thresholds can emerge; for the seesaw, this was studied recently in [42], 201].

Probably more important than the above theoretical considerations, eventually, will be the fact that the invariants are not directly measurable quantities. Conversion of the invariants into the measured sparticle masses (or extraction of relevant soft parameters from sparticle measurements) requires additional experimental input.

The prospects of measuring sparticle masses at the LHC and, possibly, at the future ILC have been studied by many authors, for a detailed review see, for example [回].

Comparing roughly the changes in spectra induced in the seesaw models studied in [43] with the changes expected in our $S O(10)$ models, we expect that a detailed, numerical calculation should be able to probe most, if not all the interesting parameter space of our models, if SUSY is found at the LHC and precise mass measurements are done with the help of an ILC.

\section{Acknowledgments}

This work is supported by the EU Network grant UNILHC PITN-GA-2009-237920 and by the Marie Curie Intra-European Fellowship within the 7th European Community Framework Programme FP7-PEOPLE-2009-IEF, contract number PIEF-GA-2009-253119. We acknowledge support from the Spanish MICINN grants FPA2008-00319/FPA and MULTIDARK CAD2009-00064 (Con-solider-Ingenio 2010 Programme) and by the Generalitat Valenciana grant Prometeo/2009/091.

\section{References}

[1] V. De Romeri, M. Hirsch, M. Malinsky, "Soft masses in SUSY SO(10) GUTs with low intermediate scales, “ Phys. Rev. D 84 (2011) 053012 [arXiv:hep-ph/1107.3412]. 
[2] A. H. Chamseddine, R. L. Arnowitt and P. Nath, “Locally Supersymmetric Grand Unification,” Phys. Rev. Lett. 49 (1982) 970.

[3] H. P. Nilles, “Supersymmetry, Supergravity And Particle Physics,” Phys. Rept. 110 (1984) 1.

[4] For a review on GMSB, see: G. F. Giudice and R. Rattazzi, "Theories with gauge-mediated supersymmetry breaking,” Phys. Rept. 322 (1999) 419 [arXiv:hep-ph/9801271].

[5] G. Weiglein et al. [LHC/LC Study Group], Phys. Rept. 426 (2006) 47 [arXiv:hep-ph/0410364].

[6] Y. Fukuda et al. [Super-Kamiokande Collaboration], Phys. Rev. Lett. 81, 1562 (1998)

[7] SNO, Q. R. Ahmad et al., Phys. Rev. Lett. 89, 011301 (2002), [nucl-ex/0204008].

[8] KamLAND, K. Eguchi et al., Phys. Rev. Lett. 90, 021802 (2003), [hep-ex/0212021].

[9] N. Arkani-Hamed and S. Dimopoulos, JHEP 0506, 073 (2005) [arXiv:hep-th/0405159].

[10] G. F. Giudice and A. Romanino, Nucl. Phys. B 699, 65 (2004) [Erratum-ibid. B 706, 65 (2005)] [arXiv:hep-ph/0406088].

[11] KamLAND Collaboration, arXiv:0801.4589 [hep-ex].

[12] For a recent review on the status of neutrino oscillation data, see: T. Schwetz, M. A. Tortola and J. W. F. Valle, New J. Phys. 10, 113011 (2008) [arXiv:0808.2016 [hep-ph]]. Version 3 on the arXive is updated with data until Feb 2010

[13] T. Schwetz, M. Tortola and J. W. F. Valle, New J. Phys. 13, 063004 (2011) [arXiv:1103.0734 [hep-ph]].

[14] E. Ma, Phys. Rev. Lett. 81, 1171 (1998) [arXiv:hep-ph/9805219].

[15] P. Minkowski, Phys. Lett. B 67 (1977) 421.

[16] T. Yanagida, in KEK lectures, ed. O. Sawada and A. Sugamoto, KEK, 1979; M Gell-Mann, P Ramond, R. Slansky, in Supergravity, ed. P. van Niewenhuizen and D. Freedman (North Holland, 1979);

[17] R.N. Mohapatra and G. Senjanovic, Phys. Rev. Lett. 44912 (1980).

[18] J. Schechter and J. W. F. Valle, Phys. Rev. D 22, 2227 (1980).

[19] T. P. Cheng and L. F. Li, Phys. Rev. D 22, 2860 (1980).

[20] R. Foot, H. Lew, X. G. He and G. C. Joshi, Z. Phys. C 44, 441 (1989).

[21] J. N. Esteves, J. C. Romao, M. Hirsch, F. Staub and W. Porod, Phys. Rev. D 83, 013003 (2011) [arXiv:1010.6000 [hep-ph]].

[22] C. Biggio and L. Calibbi, arXiv:1007.3750 [hep-ph].

[23] H. Fritzsch and P. Minkowski, Annals Phys. 93 (1975) 193.

[24] R. N. Mohapatra and J. W. F. Valle, Phys. Rev. D34, 1642 (1986).

[25] E. Akhmedov, M. Lindner, E. Schnapka and J. W. F. Valle, Phys. Rev. D53, 2752 (1996), [hep-ph/9509255]; Phys. Lett. B368, 270 (1996), [hep-ph/9507275].

[26] R. N. Mohapatra, Berlin, Germany: Springer ( 1986) 309 P. ( Contemporary Physics) 
[27] M. Cvetic and J. C. Pati, Phys. Lett. B 135, 57 (1984).

[28] R. Kuchimanchi and R. N. Mohapatra, Phys. Rev. D 48, 4352 (1993) [arXiv:hep-ph/9306290]. [29]

[29] C. S. Aulakh, K. Benakli and G. Senjanovic, Phys. Rev. Lett. 79, 2188 (1997) [arXiv:hep-ph/9703434].

[30] C. S. Aulakh, A. Melfo, A. Rasin and G. Senjanovic, Phys. Rev. D 58, 115007 (1998) [arXiv:hep-ph/9712551].

[31] J. Kopp, M. Lindner, V. Niro and T. E. J. Underwood, Phys. Rev. D 81, 025008 (2010) [arXiv:0909.2653 [hep-ph]].

[32] S. K. Majee, M. K. Parida, A. Raychaudhuri and U. Sarkar, Phys. Rev. D 75, 075003 (2007) [arXiv:hep-ph/0701109].

[33] M. Malinsky, J. C. Romao and J. W. F. Valle, Phys. Rev. Lett. 95, 161801 (2005) [arXiv:hep-ph/0506296].

[34] P. S. B. Dev and R. N. Mohapatra, Phys. Rev. D 81 (2010) 013001 [arXiv:0910.3924 [hep-ph]].

[35] K. Nakamura et al. [Particle Data Group], J. Phys. G 37, 075021 (2010).

[36] G. Aad et al. [ ATLAS Collaboration ], Phys. Lett. B700 (2011) 163-180. [arXiv:1103.6218 [hep-ex]].

[37] L. Basso, A. Belyaev, S. Moretti, G. M. Pruna and C. H. Shepherd-Themistocleous, Eur. Phys. J. C 71, 1613 (2011) [arXiv:1002.3586 [hep-ph]].

[38] S. N. Gninenko, M. M. Kirsanov, N. V. Krasnikov and V. A. Matveev, Phys. Atom. Nucl. 70 (2007) 441.

[39] A. Ferrari et al., Phys. Rev. D 62 (2000) 013001.

[40] M. R. Buckley and H. Murayama, Phys. Rev. Lett. 97, 231801 (2006) [arXiv:hep-ph/0606088].

[41] J. C. Pati and A. Salam, Phys. Rev. D 10, 275 (1974) [Erratum-ibid. D 11, 703 (1975)].

[42] M. Hirsch, S. Kaneko and W. Porod, Phys. Rev. D 78, 093004 (2008) [arXiv:0806.3361 [hep-ph]].

[43] M. Hirsch, L. Reichert and W. Porod, JHEP 1105, 086 (2011) [arXiv:1101.2140 [hep-ph]].

[44] S. P. Martin and M. T. Vaughn, Phys. Rev. D 50, 2282 (1994) [Erratum-ibid. D 78, 039903 (2008)] [arXiv:hep-ph/9311340].

[45] R. Fonseca, M. Malinsky, W. Porod and F. Staub, arXiv:1107.2670 [hep-ph].

[46] L. Lavoura, H. Kuhbock, and W. Grimus, Nucl.Phys. B754, 1 (2006), arXiv:hep-ph/0603259.

[47] M. Heinze and M. Malinsky, Phys.Rev. D83, 035018 (2011), arXiv:1008.4813.

[48] C. Amsler et al. [Particle Data Group], Phys. Lett. B 667, 1 (2008).

[49] W. Porod, Comput. Phys. Commun. 153, 275 (2003) [arXiv:hep-ph/0301101]. For the latetst version of SPheno, see the web page: http://www.physik.uni-wuerzburg.de/ porod/SPheno.html

[50] F. Staub, arXiv:0806.0538 [hep-ph]. 